GJI Seismology

\title{
Wavefront attributes in anisotropic media
}

\author{
C. Vanelle, ${ }^{1}$ I. Abakumov ${ }^{2}$ and D. Gajewski ${ }^{1}$ \\ ${ }^{1}$ Institute of Geophysics, University of Hamburg, Bundesstraße 55, 20146 Hamburg, Germany. E-mail: claudia.vanelle@uni-hamburg.de \\ ${ }^{2}$ Fachrichtung Geophysik, Freie Universität Berlin, Malteserstr. 74-100, 12249 Berlin, Germany
}

Received 2017 December 20; in original form 2017 July 18

\begin{abstract}
SUMMAR Y
Surface-measured wavefront attributes are the key ingredient to multiparameter methods, which are nowadays standard tools in seismic data processing. However, most operators are restricted to application to isotropic media. Whereas application of an isotropic operator will still lead to satisfactory stack results, further processing steps that interpret isotropic stacking parameters in terms of wavefront attributes will lead to erroneous results if anisotropy is present but not accounted for. In this paper, we derive relationships between the stacking parameters and anisotropic wavefront attributes that allow us to apply the common reflection surface type operator to 3-D media with arbitrary anisotropy for the zero-offset and finiteoffset configurations including converted waves. The operator itself is expressed in terms of wavefront attributes that are measured in the acquisition surface, that is, no model assumptions are made. Numerical results confirm that the accuracy of the new anisotropic operator is of the same magnitude as that of its isotropic counterpart.
\end{abstract}

Key words: Controlled source seismology; Seismic anisotropy; Wave propagation.

\section{INTRODUCTION}

Stacking methods have been applied in seismic data processing for the past five decades for several reasons. First, stacking results in a simulated zero-offset section, that is, a section where source and receiver coordinates coincide. Such a stacked section displays an enhanced signal-to-noise ratio compared to the original data. Furthermore, additional information is obtained in terms of the stacking parameters that relate to physical wavefield properties.

The aim of this work is the derivation of relationships between stacking parameters, that is, the values that provide the best fit of the stacking operator to the data but have no physical meaning per se, and wavefront attributes that describe physical properties of the wavefield, for example, angles and radii of curvatures of wavefronts.

In the classic common midpoint (CMP) method, stacking is carried out over offsets (Mayne 1962). The stacking parameter, assumed to coincide with the normal moveout velocity, is determined by semblance analysis (Taner \& Koehler 1969). Since the CMP concept was introduced, several extensions have been suggested to stack traces not only over offsets but also over midpoints. Carrying out such a stack in both midpoint and offset directions increases the number of traces and thus enhances the signal-to-noise ratio further. Also, the stacking surface is described by additional parameters, which, in turn, correspond to additional wavefront attributes, and therefore lead to even more information.

The most prominent examples for these multiparameter stacking operators are the common reflection surface (CRS) method (Müller 1999), multifocusing (Gelchinsky et al. 1999), and more recently non-hyperbolic CRS (Fomel \& Kazinnik 2013) and implicit CRS (Schwarz et al. 2014). Whereas the individual operators use slightly different parametrisations, they all have in common that the parameters have a physical interpretation in terms of wavefront curvatures and angles.

The wavefront attributes can be used for a variety of applications such as attribute-based time migration (Spinner 2007), multiple suppression (Dümmong \& Gajewski 2008), prestack data enhancement and regularisation (Baykulov \& Gajewski 2009), normal incidence point (NIP)-wave tomography (Duveneck 2004), diffraction imaging (Dell \& Gajewski 2011), diffraction tomography (Bauer et al. 2017) and diffraction separation (Bakhtiari Rad et al. 2018), to name but a few.

The interpretation of the stacking parameters in terms of wavefront attributes in the aforementioned operators is so far only valid in isotropic media. Therefore, attempts have been made to derive multiparameter expressions that consider anisotropy. Vanelle et al. (2012) have extended the implicit CRS operator to account for anisotropy. Since it is a 2-D formulation, their approach is restricted to a symmetry plane, but it allows otherwise for arbitrary anisotropy. In addition to the angles and wavefront curvatures used in the isotropic variant, velocity 
derivatives occur in the anisotropic case, which means that model assumptions must be made in order to apply this operator. The anisotropic CRS approximation suggested by Xu \& Stovas (2015) also depends on model assumptions.

In this manuscript, we derive relationships between the stacking parameters and the anisotropic wavefront attributes from geometrical considerations by following an approach similar to that Shah (1973) and Hubral \& Krey (1980) have applied in the isotropic case. The parameters that describe the stacking surface are, as in the isotropic case, interpreted as wavefront curvatures and angles measured in the registration surface. Since no assumptions on the model enter the derivation, it can be considered as an entirely data-driven approach.

We will show that the anisotropic zero-offset CRS operator in 2-D can be expressed in terms of four independent attributes. The shape of the operator, however, is the same as its isotropic counterpart, which allows only the determination of three parameters. In the anisotropic case, these three parameters are combinations of the anisotropic wavefront attributes. This means that for the stacking neglecting the presence of anisotropy will not compromise the result.

This changes as soon as the stacking parameters enter data processing for tasks that involve not only the computation of traveltimes but other applications of the wavefront attributes. Since the physical interpretation of the parameters is different in the presence of anisotropy, their application under the assumption of isotropy can lead to false results. For example, applications based on ray tracing that use the incidence or emergence angle to the registration surface need to distinguish between ray (group) and phase angle. Ignoring this difference, for example, for performing NIP-wave tomography (Duveneck 2004) or diffraction tomography (Bauer et al. 2017), would lead to an erroneous velocity model. During this step, model assumptions are made; however, the determination of the stacking parameters remains entirely model independent.

In many cases, it is assumed that the topmost layer under the registration surface is isotropic, where an anisotropic operator is not required. However, if we consider, for example, a downhole experiment where sources and/or receivers are located in anisotropic layers, an anisotropic operator must be used. Moreover, our new formulation provides an important step to enhance our understanding of the physics of the wavefront attributes that are nowadays an integral and established part of seismic data processing.

In this paper, we begin with the derivation of the anisotropic CRS operator for the zero-offset situation for monotypic waves in 2-D. In Section 2.2, we extend the operator to the general finite-offset case that is also pertinent for converted waves. We consider the 3-D case for arbitrary anisotropy and wave type in an appendix. We demonstrate the accuracy of the new operator with numerical examples in 2-D and 3-D. The following discussion and conclusions bring our work to a close.

\section{METHOD}

The CRS operator was introduced by Müller (1999). It describes a hyperbolic traveltime surface in midpoint and half-offset coordinates that is expressed in terms of kinematic wavefield properties or attributes, namely angles and wavefront curvatures, under the assumption of isotropy. In this section, we derive a corresponding anisotropic operator. Our derivation follows the geometrical approach by Shah (1973) and Hubral \& Krey (1980); however, we assume the medium under consideration to be anisotropic. This will lead us to a new CRS operator for monotypic waves in the zero-offset situation, beginning with the 2-D case. In a second step, we introduce a corresponding finite-offset CRS operator by extending the results derived for the zero-offset case in conjunction with a traveltime expression introduced by Vanelle \& Gajewski (2002). The resulting finite-offset operator is applicable to monotypic and converted waves in any medium where wave propagation takes place in a 2-D plane, for example, VTI, HTI, TTI media as well as in the symmetry planes of orthorhombic media. In the Appendix, we generalise our results to obtain a 3-D finite-offset operator for arbitrary anisotropy and wave type.

\subsection{Anisotropic CRS for 2-D zero offset}

Assuming that the traveltime function is smooth and at least twice differentiable, it can be expanded into a Taylor series. We carry out the expansion in midpoint $\left(x_{m}\right)$ and half-offset $(h)$ coordinates until second order for an expansion point at $x_{m}=x_{0}$ and $h=0$, that is, for zero offset,

$t=t_{0}+\frac{\partial t}{\partial x_{m}} \Delta x_{m}+\frac{\partial t}{\partial h} h+\frac{1}{2} \frac{\partial^{2} t}{\partial x_{m}^{2}} \Delta x_{m}^{2}+\frac{1}{2} \frac{\partial^{2} t}{\partial h^{2}} h^{2}+\frac{\partial^{2} t}{\partial x_{m} \partial h} \Delta x_{m} h$,

where $\Delta x_{m}=x_{m}-x_{0}$. Keeping reciprocity in mind, that is, the symmetry of the wavefield response, and therefore the traveltime, with regard to interchanging the source and receiver position, the following derivatives must vanish:

$\frac{\partial t}{\partial h}=0 \quad$ and $\quad \frac{\partial^{2} t}{\partial x_{m} \partial h}=0$.

In conclusion, the traveltime expression 1 reduces to

$t=t_{0}+\frac{\partial t}{\partial x_{m}} \Delta x_{m}+\frac{1}{2} \frac{\partial^{2} t}{\partial x_{m}^{2}} \Delta x_{m}^{2}+\frac{1}{2} \frac{\partial^{2} t}{\partial h^{2}} h^{2}$.

This is a parabolic expression. It is, however, known that reflection traveltimes are better approximated by hyperbolic than parabolic operators (e.g. Ursin 1982; Gjøystdal et al. 1984). To obtain a hyperbolic expression like the original isotropic CRS operator, we square both sides of 


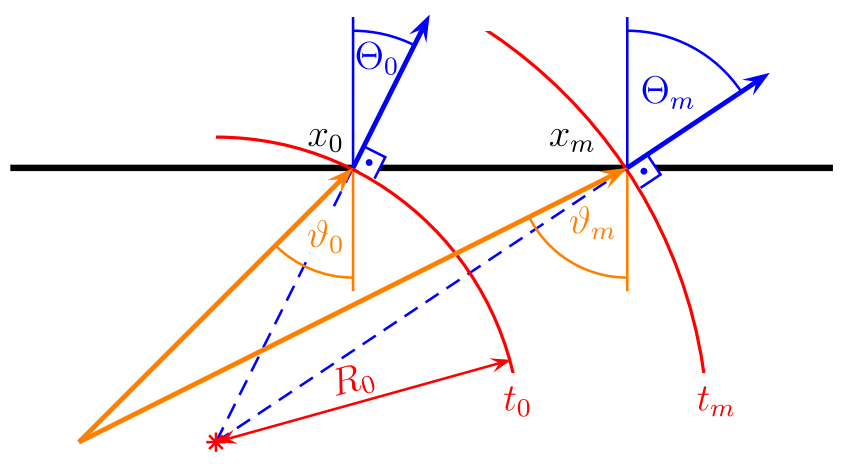

Figure 1. Rays (orange), slowness directions (blue) and their corresponding angles at $x_{0}$ and $x_{m}$. Wavefronts (red) at the times $t_{0}$ (with radius $R_{0}$ ) and $t_{m}$ are locally approximated by circles. Unlike the rays, the slownesses are perpendicular to the wavefronts. The red star marks the hypothetic centre of the wavefront.

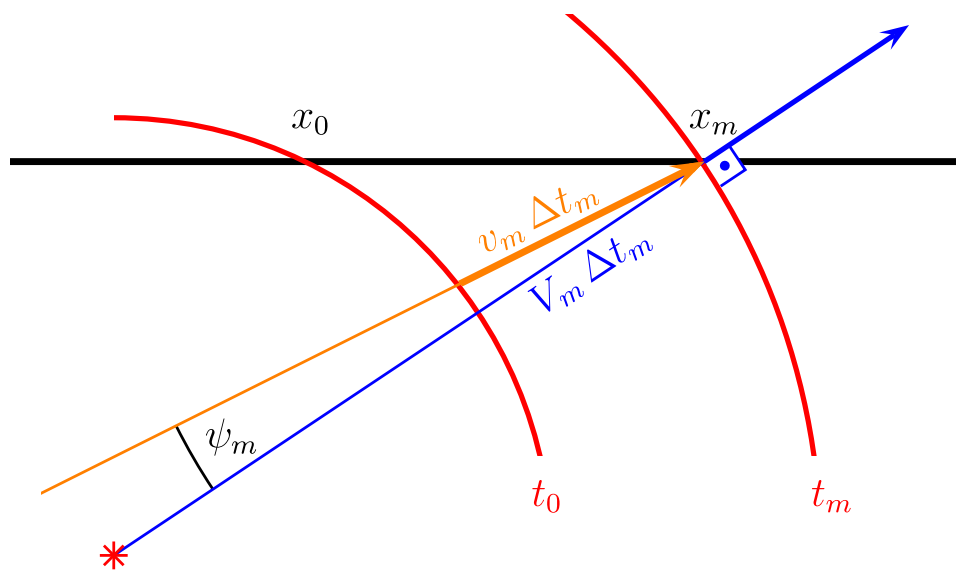

Figure 2. Distances between the wavefronts at $x_{0}$ and $x_{m}$ expressed by the phase and group velocities $V_{m}$ and $v_{m}$, respectively, and the traveltime difference, that is, moveout, $\Delta t=t_{m}-t_{0}$. The angle $\psi_{m}$ lies between the slowness and ray direction. It relates the phase and group velocities by $V_{m}=v_{m} \cos \psi_{m}$.

eq. (3) and omit terms of order higher than two, which leads to

$t^{2}=\left(t_{0}+\frac{\partial t}{\partial x_{m}} \Delta x_{m}\right)^{2}+t_{0} \frac{\partial^{2} t}{\partial x_{m}^{2}} \Delta x_{m}^{2}+t_{0} \frac{\partial^{2} t}{\partial h^{2}} h^{2}$.

Our aim is now to express the remaining derivatives in terms of physically intuitive wavefront attributes like in Müller's (1999) result for the isotropic case.

In the following, ray velocities are denoted by $v$, phase velocities by $V$ and slowness vectors by $\boldsymbol{p}$. A subscript 0 denotes a quantity taken at the expansion point, $x_{0}$, and subscript $m$ indicates a quantity taken at a position $x_{m}$. Note that, unlike in commonly-used notation, $v_{0}$ and $V_{0}$ are not velocities in vertical direction of propagation but velocities taken in the expansion point $x_{0}$ associated with the ray and slowness direction at $x_{0}$, respectively. Furthermore, angles $\vartheta$ are ray (group) angles and $\Theta$ are phase angles. Angles $\psi=\vartheta-\Theta$ lie between phase and ray direction.

Fig. 1 displays a sketch of two zero-offset rays and their associated wavefronts arriving at locations $x_{m}$ and $x_{0}$ in the registration surface. In addition to the ray angles $\vartheta_{m}$ and $\vartheta_{0}$, the figure shows the radius of curvature of the incident wavefront at $x_{0}$, denoted $R_{0}$. The horizontal slownesses corresponding to the rays are $p_{m}=\sin \Theta_{m} / V_{m}$ and $p_{0}=\sin \Theta_{0} / V_{0}$. The ray and slowness at $x_{m}$ are depicted in Fig. 2, where those at $x_{0}$ were omitted for simplicity.

For our derivation, we assume that all distances are infinitesimal. We additionally assume that the medium is anisotropic but homogeneous within the considered vicinity. In particular, this means that the ray (group) and phase directions do not change along the ray. Figs $1,2,3$, 4 are drawn under this assumption. Furthermore, the operator derived in this section is a 2-D expression. Therefore, waves are assumed to propagate in the $x-z$ plane.

In order to relate the derivatives in the Taylor expansion 4 to the quantities we just introduced, we use the law of sines for the triangle in Fig. 3. The angles and the lengths of their opposing sides define the geometry we need for the sought-for relation. They are

(1) the angle $90^{\circ}-\vartheta_{m}$ and the distance between the two rays, approximated by the arclength $R_{0} \Delta \Theta_{m}=R_{0}\left(\Theta_{m}-\Theta_{0}\right)$ (note that the arclength is calculated by the phase angle difference and not by the difference between ray angles because unlike the slowness vectors, the rays are not perpendicular to the wavefronts, see Fig. 4),

(2) the angle $\Theta_{0}+\Delta \Theta_{m} / 2$ and the distance $v_{m} \Delta t_{m}=v_{m}\left(t_{m}-t_{0}\right)=v_{m}\left(t\left(x_{m}\right)-t\left(x_{0}\right)\right)$, 


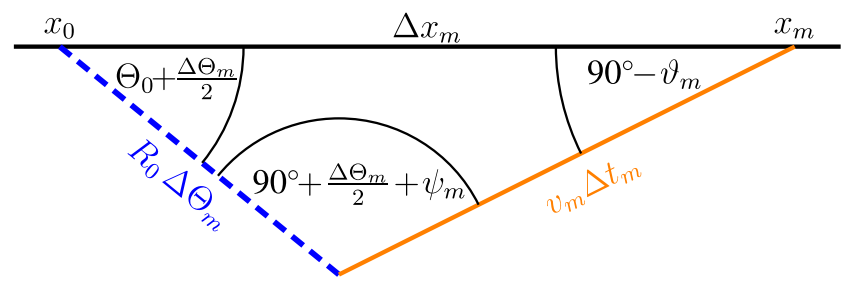

Figure 3. Triangle for the law of sines (see the text). The additional contribution of $\Delta \Theta_{m} / 2$ results from the projection of the wavefront element onto the straight line $R_{0} \Delta \Theta_{m}$.

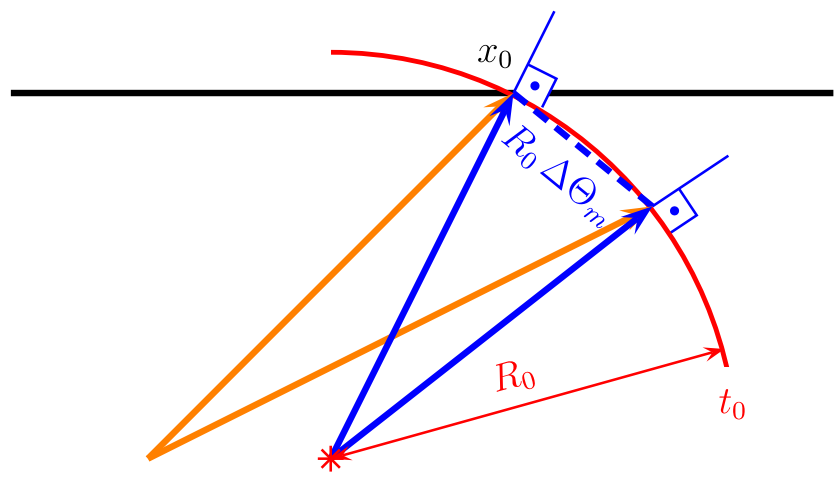

Figure 4. The wavefront segment at $x_{0}$ that corresponds to the arc between $\Theta_{m}$ and $\Theta_{0}$ is approximated by the dashed line. Its length is given by $R_{0} \Delta \Theta_{m}$, where $\Delta \Theta_{m}=\Theta_{m}-\Theta_{0}$.

(3) the angle $90^{\circ}+\psi_{m}+\Delta \Theta_{m} / 2$ and the distance $\Delta x_{m}=x_{m}-x_{0}$.

Using these, the law of sines provides the following relationships:

$\frac{\sin \left(90^{\circ}+\psi_{m}+\Delta \Theta_{m} / 2\right)}{\Delta x_{m}}=\frac{\sin \left(\Theta_{0}+\Delta \Theta_{m} / 2\right)}{v_{m} \Delta t_{m}}=\frac{\sin \left(90^{\circ}-\vartheta_{m}\right)}{R_{0} \Delta \Theta_{m}}$,

or, applying $\sin \left(90^{\circ} \pm \alpha\right)=\cos \alpha$,

$\frac{\cos \left(\psi_{m}+\Delta \Theta_{m} / 2\right)}{\Delta x_{m}}=\frac{\sin \left(\Theta_{0}+\Delta \Theta_{m} / 2\right)}{v_{m} \Delta t_{m}}=\frac{\cos \vartheta_{m}}{R_{0} \Delta \Theta_{m}}$.

With

$\frac{\Delta t_{m}}{\Delta x_{m}}=\frac{\sin \left(\Theta_{0}+\Delta \Theta_{m} / 2\right)}{v_{m} \cos \left(\psi_{m}+\Delta \Theta_{m} / 2\right)}$

we obtain

$\left.\frac{\partial t_{m}}{\partial x_{m}}\right|_{x_{0}}=\lim _{x_{m} \rightarrow x_{0}} \frac{\Delta t_{m}}{\Delta x_{m}}=\frac{\sin \Theta_{0}}{v_{0} \cos \psi_{0}}$.

Substituting the relationship between phase and group velocity, $V=v \cos \psi$ (e.g. Tsvankin 2001, see also Fig. 2), we find that this derivative is, as expected, the horizontal slowness $p_{0}$ at $x_{0}$,

$p_{0}=\left.\frac{\partial t_{m}}{\partial x_{m}}\right|_{x_{0}}=\frac{\sin \Theta_{0}}{V_{0}}$.

Taking into account that the situation depicted in Figs 1 and 4 that led us to this result is a one-way process, whereas the Taylor expansion in eq. (4) is a two-way process, we find the first-order derivative in eq. (4) to be

$\left.\frac{\partial t}{\partial x_{m}}\right|_{x_{0}, h=0}=\left.2 \frac{\partial t_{m}}{\partial x_{m}}\right|_{x_{0}}=2 \frac{\sin \Theta_{0}}{V_{0}}$.

For the determination of the second-order derivative of the traveltime with respect to $x_{m}$, we differentiate the general expression for the horizontal slowness, $p=\sin \Theta / V$, that is,

$\left.\frac{\partial^{2} t_{m}}{\partial x_{m}^{2}}\right|_{x_{0}}=\left.\frac{\partial p_{m}}{\partial x_{m}}\right|_{x_{0}}=\left[\frac{1}{V_{m}^{2}}\left(V_{m} \frac{\partial \sin \Theta_{m}}{\partial x_{m}}-\sin \Theta_{m} \frac{\partial V_{m}}{\partial x_{m}}\right)\right]_{x_{0}}=\left[\frac{1}{V_{m}^{2}}\left(V_{m} \cos \Theta_{m} \frac{\partial \Theta_{m}}{\partial x_{m}}-\sin \Theta_{m} \frac{\partial V_{m}}{\partial \Theta_{m}} \frac{\partial \Theta_{m}}{\partial x_{m}}\right)\right]_{x_{0}}$.

This expression contains two unknown partial derivatives. One of them can be resolved by the relationship between the phase velocity and the vertical component $v_{z}$ of the group velocity. With (e.g. Tsvankin 2001)

$v_{z}=v \cos \vartheta=V \cos \Theta-\frac{\partial V}{\partial \Theta} \sin \Theta$ 


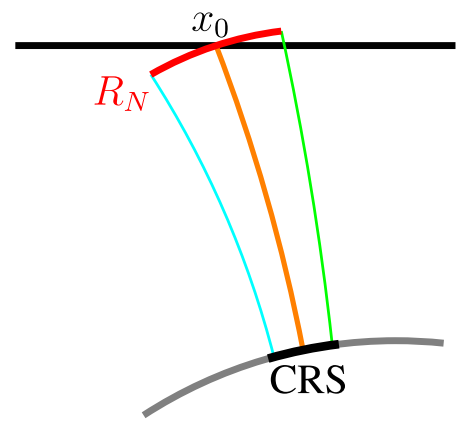

(a)

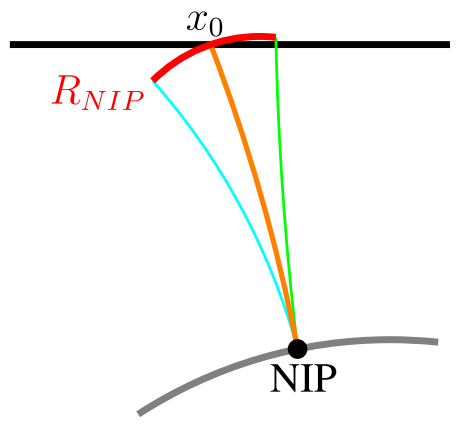

(b)

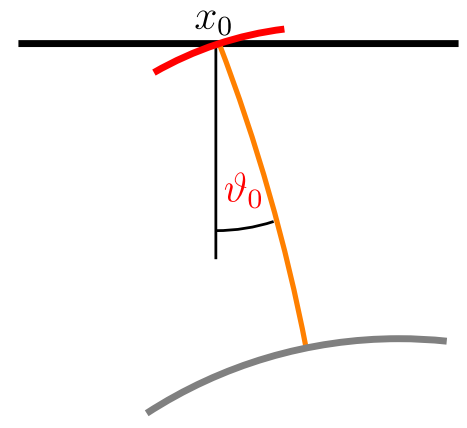

(c)

Figure 5. (a) Hypothetic zero-offset experiment: radius of curvature $R_{N}$, measured at $x_{0}$, of a wavefront (red) emitted by an exploding curved reflector element (black) denoted CRS (common reflection surface). (b) Hypothetic CMP experiment: radius of curvature $R_{N I P}$, measured at $x_{0}$, of a wavefront (red) emitted by a point source (black) in the NIP (normal incidence point). A central ray (orange) and two paraxial rays (cyan and green) are also shown. Note that the zero-offset rays are only perpendicular to the reflector (grey) in the isotropic case. In the anisotropic case, normal incidence refers to the slowness, not the ray direction. In the special case of a point diffractor, the radii $R_{N}$ and $R_{N I P}$ coincide. (c) The ray angle $\vartheta_{0}$ lies between the central ray and the vertical direction.

we find that

$\frac{\partial V}{\partial \Theta}=\frac{V \cos \Theta-v \cos \vartheta}{\sin \Theta}$.

For the remaining unknown partial derivative in eq. (11), we can again apply the law of sines 6 , where

$\frac{\Delta \Theta_{m}}{\Delta x_{m}}=\frac{\cos \vartheta_{m}}{R_{0} \cos \left(\psi_{m}+\Delta \Theta_{m} / 2\right)}$

leads to

$\left.\frac{\partial \Theta_{m}}{\partial x_{m}}\right|_{x_{0}}=\lim _{x_{m} \rightarrow x_{0}} \frac{\Delta \Theta_{m}}{\Delta x_{m}}=\frac{\cos \vartheta_{0}}{R_{0} \cos \psi_{0}}$.

Substituting eqs (13) and (15) into eq. (11), the second-order derivative (11) becomes

$\left.\frac{\partial^{2} t_{m}}{\partial x_{m}^{2}}\right|_{x_{0}}=\frac{\cos ^{2} \vartheta_{0}}{v_{0} \cos ^{3} \psi_{0} R_{0}}$,

and, therefore,

$\left.\frac{\partial^{2} t}{\partial x_{m}^{2}}\right|_{x_{0}, h=0}=\left.2 \frac{\partial^{2} t_{m}}{\partial x_{m}^{2}}\right|_{x_{0}}=2 \frac{\cos ^{2} \vartheta_{0}}{v_{0} \cos ^{3} \psi_{0} R_{0}}$.

Note that this second-order derivative can also be expressed by the phase angle and phase velocity; however, the expression is more compact in terms of ray/group properties.

Before we turn to the derivation of the remaining term in the Taylor expansion 4, let us take a closer look at the wavefront curvature radii displayed in Fig.5. In Fig. 5(a), $R_{N}$ describes a fictitious wavefront that is generated by an exploding reflector element, the so-called common reflection surface. It is commonly referred to as the normal wavefront (Tygel et al. 1997) and measured by the zero-offset experiment also described in Fig. 5(a). We recognise that the radius of curvature $R_{0}$ coincides with $R_{N}$. The second-order traveltime derivative 17 is therefore equal to

$$
\left.\frac{\partial^{2} t}{\partial x_{m}^{2}}\right|_{x_{0}, h=0}=\left.2 \frac{\partial^{2} t_{m}}{\partial x_{m}^{2}}\right|_{x_{0}}=2 \frac{\cos ^{2} \vartheta_{0}}{v_{0} \cos ^{3} \psi_{0} R_{N}} .
$$

If we now consider a common-midpoint (CMP) experiment, we find a corresponding fictitious wavefront, the NIP wavefront generated by a point source in the NIP as depicted in Fig.5(b). As for the derivation of the second-order derivative with respect to $x_{m}$, that is, for the zero-offset experiment, we can follow the same steps for the second-order derivative with respect to $h$. In the latter case, we have the CMP experiment, and all we need to do is replace $R_{0}$ in eq. (17) with the radius of the NIP wavefront, $R_{N I P}$, and $x_{m}$ with $h$. We obtain

$$
\left.\frac{\partial^{2} t}{\partial h^{2}}\right|_{x_{0}, h=0}=2 \frac{\cos ^{2} \vartheta_{0}}{v_{0} \cos ^{3} \psi_{0} R_{N I P}} \text {. }
$$

In conclusion, the anisotropic CRS operator for the zero-offset case is given by

$t^{2}=\left(t_{0}+2 \frac{\sin \Theta_{0}}{V_{0}} \Delta x_{m}\right)^{2}+2 t_{0} \frac{\cos ^{2} \vartheta_{0}}{v_{0} \cos ^{3} \psi_{0}}\left(\frac{\Delta x_{m}^{2}}{R_{N}}+\frac{h^{2}}{R_{N I P}}\right)$. 
We elaborate on the implications of this result in Section 4. In the following section, we use eq. (20) and the work by Vanelle \& Gajewski (2002) to derive the extension to the finite-offset situation.

\subsection{Anisotropic CRS for 2-D finite offset}

In order to derive the anisotropic finite-offset operator, we use the traveltime for an arbitrary source-receiver combination $(s, g)$ in the vicinity of an expansion point at $\left(s_{0}, g_{0}\right)$ in the hyperbolic expression suggested by Vanelle \& Gajewski (2002),

$t^{2}(s, g)=\left(t_{0}+p_{g} \Delta g-p_{s} \Delta s\right)^{2}+t_{0}\left(G \Delta g^{2}-S \Delta s^{2}-2 N \Delta s \Delta g\right)$

where $\Delta s=s-s_{0}$ and $\Delta g=g-g_{0}$ are the distances of the source and receiver positions to the expansion point, respectively, and $t_{0}$ is the traveltime in the expansion point, that is, $t_{0}=t\left(s_{0}, g_{0}\right)$. The coefficients in eq. (21) are the first- and second-order derivatives of the traveltime with respect to source and receiver coordinates. Namely, the first-order derivatives,

$p_{s}=-\left.\frac{\partial t}{\partial s}\right|_{s_{0}, g_{0}}$ and $\quad p_{g}=\left.\frac{\partial t}{\partial g}\right|_{s_{0}, g_{0}}$,

are the horizontal slownesses at the source and receiver, respectively. The second-order derivatives are given by

$S=-\left.\frac{\partial^{2} t}{\partial s^{2}}\right|_{s_{0}, g_{0}}, \quad G=\left.\frac{\partial^{2} t}{\partial g^{2}}\right|_{s_{0}, g_{0}} \quad, \quad$ and $\quad N=-\left.\frac{\partial^{2} t}{\partial s \partial g}\right|_{s_{0}, g_{0}}$.

In midpoint and half-offset coordinates $\left(x_{m}, h\right)$, with $x_{m}=(g+s) / 2, x_{0}=\left(g_{0}+s_{0}\right) / 2, h=(g-s) / 2, h_{0}=\left(g_{0}-s_{0}\right) / 2$ and the corresponding distances $\Delta h=h-h_{0}, \Delta x_{m}=x_{m}-x_{0}$, eq. (21) reads

$t^{2}\left(x_{m}, h\right)=\left(t_{0}+\left(p_{g}-p_{s}\right) \Delta x_{m}+\left(p_{g}+p_{s}\right) \Delta h\right)^{2}+t_{0}\left((G-S-2 N) \Delta x_{m}^{2}+(G-S+2 N) \Delta h^{2}+2(G+S) \Delta x_{m} \Delta h\right)$.

Application of eq. (24) as stacking operator leads to the determination of $\left(p_{g} \pm p_{s}\right),(G-S \pm 2 N)$ and $(G+S)$, and thus to the parameters $p_{g}, p_{s}, S, G, N$.

Again, in the zero-offset situation for monotypic waves, all traveltime expressions given above must be symmetric with respect to interchanging the source and receiver, that is, changing the sign of $h$. Furthermore, we have $h_{0}=0$ and $\Delta h=h$. Therefore, the following relations apply:

$p_{g}=-p_{s} \quad$ and $\quad G=-S$.

In conclusion, eq. (24) simplifies. It can be expressed either in terms of $\left(p_{s}, S, N\right)$ or $\left(p_{g}, G, N\right)$, that is,

$t^{2}\left(x_{m}, h\right)=\left(t_{0}-2 p_{s} \Delta x_{m}\right)^{2}+2 t_{0}\left((-S-N) \Delta x_{m}^{2}+(-S+N) h^{2}\right)$

or

$t^{2}\left(x_{m}, h\right)=\left(t_{0}+2 p_{g} \Delta x_{m}\right)^{2}+2 t_{0}\left((G-N) \Delta x_{m}^{2}+(G+N) h^{2}\right)$.

We now consider the zero-offset CRS operator, eq. (20). Comparing the coefficients in eqs (26) and (27) with those in eq. (20), we find that the wavefront attributes in eq. (20) and the parameters in eqs (26) and/or (27) are related by

$$
\begin{aligned}
\frac{\cos ^{2} \vartheta_{0}}{v_{0} \cos ^{3} \psi_{0} R_{N}} & =G-N=-S-N, \\
\frac{\cos ^{2} \vartheta_{0}}{v_{0} \cos ^{3} \psi_{0} R_{N I P}}=G+N & =-S+N, \\
\frac{\sin \Theta_{0}}{V_{0}} & =p_{g}=-p_{s} .
\end{aligned}
$$

In a formally identical fashion to the zero-offset attributes in eq. (28), we introduce finite-offset attributes. These are, like those in the zero-offset case, angles and radii of curvatures of fictitious wavefronts (see Fig.6):

$$
\begin{aligned}
\frac{\cos ^{2} \vartheta_{g}}{v_{g} \cos ^{3} \psi_{g} R_{g}^{C O}} & =G-N, & \frac{\cos ^{2} \vartheta_{s}}{v_{s} \cos ^{3} \psi_{s} R_{s}^{C O}} & =-S-N, \\
\frac{\cos ^{2} \vartheta_{g}}{v_{g} \cos ^{3} \psi_{g} R_{g}^{C M P}} & =G+N, & \frac{\cos ^{2} \vartheta_{s}}{v_{s} \cos ^{3} \psi_{s} R_{s}^{C M P}} & =-S+N, \\
\frac{\sin \Theta_{g}}{V_{g}} & =p_{g}, & \frac{\sin \Theta_{s}}{V_{s}} & =-p_{s} .
\end{aligned}
$$




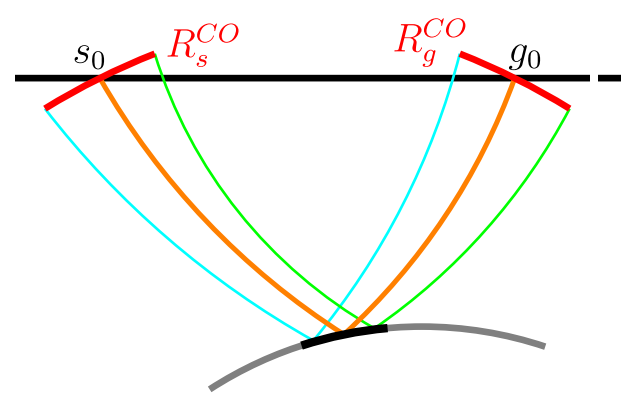

(a)

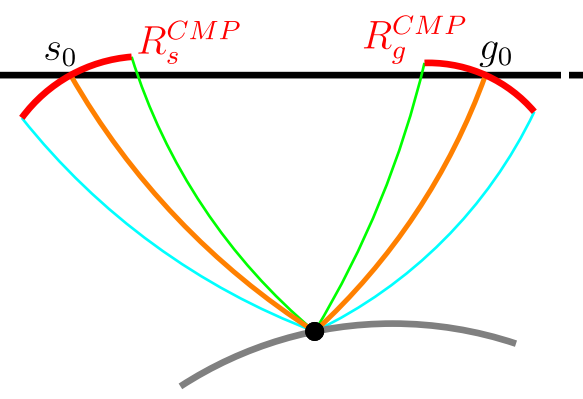

(b)

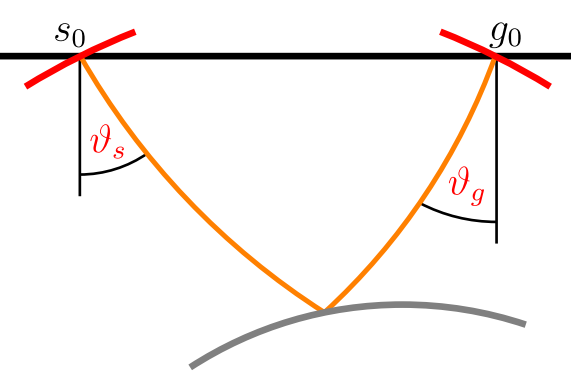

(c)

Figure 6. (a) Hypothetic finite-offset experiment: Radii of curvature $R_{s}^{C O}$ and $R_{g}^{C O}$, measured at $s_{0}$ and $g_{0}$, respectively, of wavefronts (red) reflected by a curved reflector element (black). (b) Hypothetic CMP experiment: radii of curvature $R_{s}^{C M P}$ and $R_{g}^{C M P}$, measured at $s_{0}$ and $g_{0}$, respectively, of wavefronts (red) associated with a common reflection point (black). A central ray (orange) and two paraxial rays (cyan and green) are also shown. (c) The ray angles $\vartheta_{s}$ and $\vartheta_{g}$, respectively, lie between the central ray and the vertical direction.

In contrast to the zero-offset case, the phase directions are no longer normal to the interface, and therefore a normal incidence point does not exist. Furthermore, the zero-offset attributes describe a one-way process. In the finite-offset case, where the downgoing and upgoing rays no longer coincide, a two-way process has to be considered. Our choice of attributes accounts for this fact. In conclusion, our operator reads

$$
\begin{aligned}
& t^{2}\left(x_{m}, h\right)=\left[t_{0}+\left(\frac{\sin \Theta_{g}}{V_{g}}+\frac{\sin \Theta_{s}}{V_{s}}\right) \Delta x_{m}+\left(\frac{\sin \Theta_{g}}{V_{g}}-\frac{\sin \Theta_{s}}{V_{s}}\right) \Delta h\right]^{2}+t_{0}\left[\frac{\cos ^{2} \vartheta_{g}}{v_{g} \cos ^{3} \psi_{g} R_{g}^{C O}}+\frac{\cos ^{2} \vartheta_{s}}{v_{s} \cos ^{3} \psi_{s} R_{s}^{C O}}\right] \Delta x_{m}^{2} \\
& +t_{0}\left[\frac{\cos ^{2} \vartheta_{g}}{v_{g} \cos ^{3} \psi_{g} R_{g}^{C M P}}+\frac{\cos ^{2} \vartheta_{s}}{v_{s} \cos ^{3} \psi_{s} R_{s}^{C M P}}\right] \Delta h^{2}+2 t_{0}\left[\frac{\cos ^{2} \vartheta_{g}}{v_{g} \cos ^{3} \psi_{g} R_{g}^{C M P}}-\frac{\cos ^{2} \vartheta_{s}}{v_{s} \cos ^{3} \psi_{s} R_{s}^{C M P}}\right] \Delta x_{m} \Delta h .
\end{aligned}
$$

Introducing the notation

$$
\begin{gathered}
M_{g}^{C O}=G-N, M_{s}^{C O}=S+N, \\
M_{g}^{C M P}=G+N, M_{s}^{C M P}=S-N
\end{gathered}
$$

and using eq. (29) we can write eq. (30) as

$$
\begin{aligned}
t^{2}\left(x_{m}, h\right)= & {\left[t_{0}+\left(p_{g}-p_{s}\right) \Delta x_{m}+\left(p_{g}+p_{s}\right) \Delta h\right]^{2}+t_{0}\left[M_{g}^{C O}-M_{s}^{C O}\right] \Delta x_{m}^{2}+t_{0}\left[M_{g}^{C M P}-M_{s}^{C M P}\right] \Delta h^{2} } \\
& +2 t_{0}\left[M_{g}^{C M P}+M_{s}^{C M P}\right] \Delta x_{m} \Delta h .
\end{aligned}
$$

Note that the mixed term in eqs (30) and (32) could also be expressed by the $R_{s, g}^{C O}$ or $M_{s, g}^{C O}$ because solving eq. (31) for $N$ leads to

$M_{g}^{C M P}+M_{s}^{C M P}=M_{g}^{C O}+M_{s}^{C O}$.

However, it has been observed in the isotropic zero-offset case that the parameter determination leads to less stable results for $R_{N}$ than for $R_{N I P}$ (see, e.g. Walda \& Gajewski 2015). This observation also applies to the $R_{s, g}^{C O}$ because these correspond to $R_{N}$ in the zero-offset case. Consequently, we prefer to express the mixed term in eq. (30) by the attributes $R_{s, g}^{C M P}$. In practice, midpoint apertures, that is, distances $\Delta x_{m}$, are chosen small. Therefore, the potential instability of $R_{s, g}^{C O}$ has limited impact on the stack since it occurs only in the second-order term for $\Delta x_{m}^{2}$.

For applications like NIP-wave and diffraction tomography (Duveneck 2004; Bauer et al. 2017), $R_{s, g}^{C O}$ are not required. If all attributes in eq. (32) are determined, the equality 33 could be used as a means for quality control for the attributes $R_{s, g}^{C O}$.

A finite-offset operator with according attributes for the isotropic case was introduced by Zhang et al. (2001). The wavefront curvature radii $R_{s, g}^{C M P}$ and $R_{s, g}^{C O}$ in eq. (29) were adopted from that work, where their names refer to the CMP and common-offset (CO) experiment, respectively. The operator by Zhang et al. (2001) is expressed by the two curvatures $K_{s, g}^{C M P}=1 / R_{s, g}^{C M P}$ and a third wavefront curvature, $K^{C S}$ that is measured at the receiver in a common-source (CS) experiment. Their expression, however, does not exhibit the symmetry of the attributes that our expression displays. Furthermore, the use of $R_{s, g}^{C O}$ as opposed to $K^{C S}$ avoids potential issues arising from the reflection point dispersal that always occurs in a common-source experiment. Owing to these reasons, we prefer to express the anisotropic operator as given by eq. (32).

The derivation of the extension of the anisotropic finite-offset CRS operator 32 to the general 3-D case is given in the Appendix.

In the following section, we demonstrate our results with numerical examples. 
Relative traveltime errors for a circular reflector

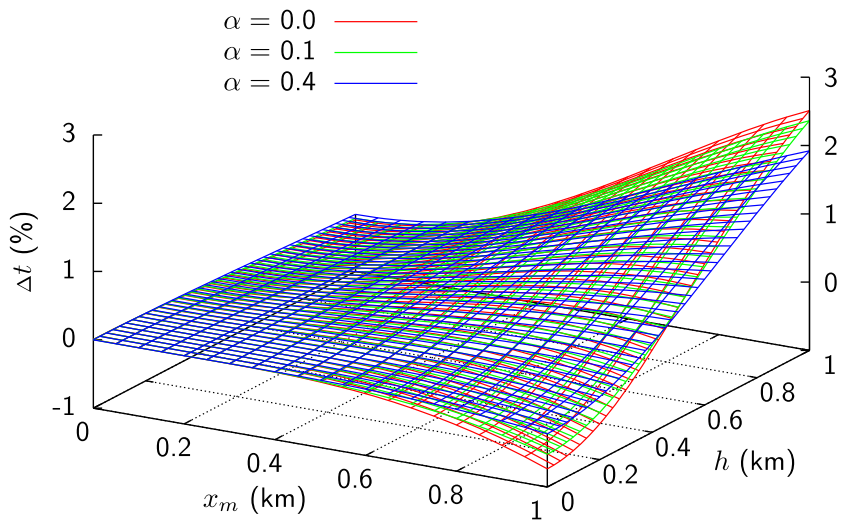

Figure 7. Relative traveltime errors of the anisotropic zero-offset CRS operator for a circular reflector with a radius of $1 \mathrm{~km}$ in a homogeneous medium with elliptical anisotropy. The CMP under consideration is located at $x_{0}=0.2 \mathrm{~km}$.

\section{NUMERICAL EXAMPLES}

In this section, we demonstrate the accuracy of the 2-D and 3-D zero-offset operators by comparing the resulting traveltimes to reference values. Since the performance of their isotropic counterparts is well established and the shape of the operator is the same, we have decided to restrict the examination to examples with a circular reflector and a point diffractor in a homogeneous anisotropic medium with elliptical symmetry in 2-D and a complex-shaped reflector under a homogeneous anisotropic overburden with ellipsoidal symmetry in 3-D.

\subsection{2-D zero-offset example}

For our 2-D example, we have chosen a medium with elliptical symmetry because this is the only anisotropic medium where analytical solutions exist for the velocities as well as for the angles and wavefront curvatures.

It has been shown for the isotropic CRS that its accuracy decreases with increasing reflector curvature (e.g. Vanelle et al. 2010). It performs worst for diffractions because diffraction traveltimes are better described by a double square root expression than by a hyperbola. We found the same general behaviour also for the anisotropic CRS. Since it can be shown that the operator 32 coincides with the exact traveltime for the case of an inclined or horizontal planar reflector in a homogeneous background medium with elliptical anisotropy, we present only results for a circular reflector and for a point diffractor.

The centre of the circular reflector with a radius of $1 \mathrm{~km}$ is located at $(0 ; 2) \mathrm{km}$. The point diffractor is located at $(0 ; 1) \mathrm{km}$. In order to quantify the anisotropy, we use a parameter $\alpha$ as follows:

(1) for $q \mathrm{P}$-waves $\alpha=\epsilon=\delta$, where $\epsilon$ and $\delta$ are Thomsen's parameters (Thomsen 1986),

(2) for SH-waves $\alpha=\gamma$, where $\gamma$ is Thomsen's parameter (Thomsen 1986),

(3) for $q \mathrm{SV}$-waves $\alpha=0$.

The medium has a vertical velocity of $v_{z}=2 \mathrm{~km} \mathrm{~s}^{-1}$ and we have considered values for $\alpha=0$ (i.e. isotropy), $\alpha=0.1$ (weak anisotropy) and $\alpha=0.4$. Reference traveltimes were generated using the NORSAR ray modelling software for the reflections and analytically, following Daley \& Hron (1979a,b), for the diffractions. The expansion point was taken at $x_{0}=0.2 \mathrm{~km}$ in all cases.

Fig. 7 shows the relative errors of the reflection traveltime for midpoint deviations of up to $0.8 \mathrm{~km}$ and an offset-to-target ratio of two. Keeping in mind that in stacking, midpoint deviations as well as offset-to-target ratios are in practice smaller than the shown range, we find the resulting accuracy, that is, the magnitude of traveltime errors, satisfying. It is interesting to note that with increasing degree of anisotropy, the accuracy improves slightly. We attribute this observation to the fact that the reflection traveltimes resulting from the circular interface are not hyperbolic, and it appears that the traveltimes in the anisotropic case provide a better fit to the hyperbolic shape of the underlying operator. Overall, both the isotropic and the anisotropic example display the same magnitude of accuracy.

In the diffraction case displayed in Fig. 8, we observe that, as expected, the accuracy is slightly lower than for the reflection. Again, the accuracy for the anisotropic examples is slightly higher than for the isotropic case. The overall accuracy is high also for the diffraction example.

\subsection{3-D zero-offset example}

For our 3-D example, we have chosen a complex-shaped reflector under a homogeneous ellipsoidally anisotropic overburden. The motivation for choosing this model was the investigation of the operator's accuracy as a function of azimuth. Fig. 9 shows the model. The horizontal 
Relative traveltime errors for a point diffractor

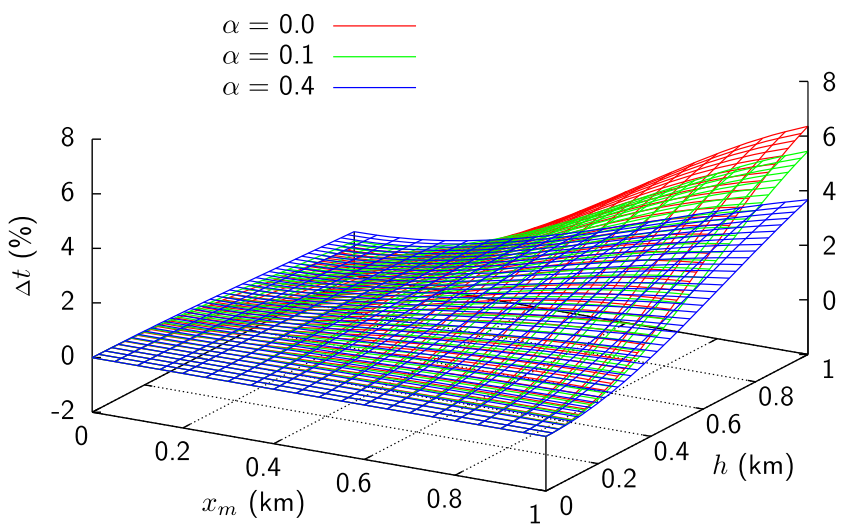

Figure 8. Relative traveltime errors of the anisotropic zero-offset CRS operator for a point diffractor in a homogeneous medium with elliptical anisotropy. The CMP under consideration is located at $x_{0}=0.2 \mathrm{~km}$.

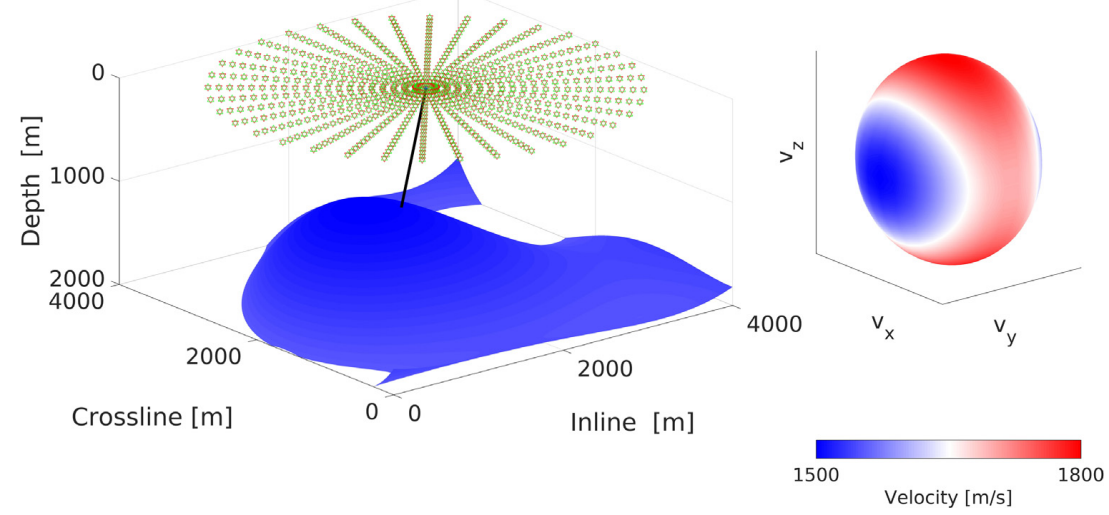

Figure 9. Velocity model for the 3-D example featuring a homogeneous overburden with ellipsoidal symmetry over a complex-shaped reflector. Red and green triangles mark the source and receiver positions. The black line corresponds to the zero-offset ray under consideration.
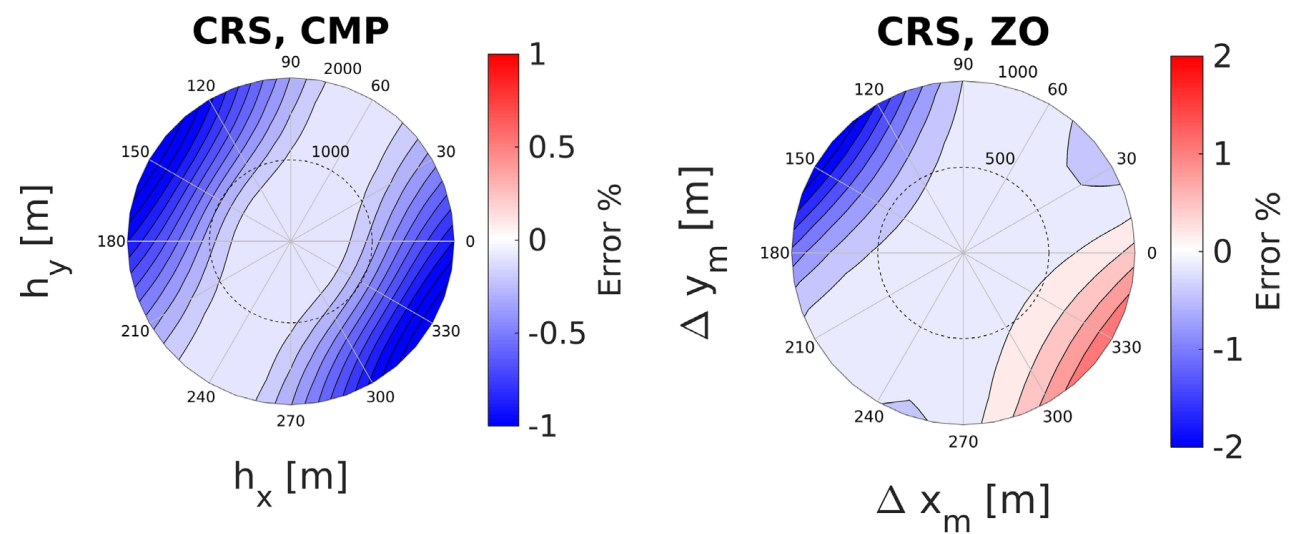

Figure 10. Relative traveltime errors of the anisotropic zero-offset CRS operator for a complex-shaped reflector under a homogeneous overburden with ellipsoidal anisotropy for (a) $\Delta \boldsymbol{x}_{\boldsymbol{m}}=0$, that is, CMP traveltimes; (b) $\Delta \boldsymbol{h}=0$, that is, zero-offset traveltimes.

and vertical velocities, respectively, are $v_{x}=1.5 \mathrm{~km} \mathrm{~s}^{-1}, v_{y}=1.7 \mathrm{~km} \mathrm{~s}^{-1}, v_{z}=1.8 \mathrm{~km} \mathrm{~s}^{-1}$. Fig. 10 displays the resulting relative traveltime errors. As for the 2-D examples, we find that the 3-D operator provides a highly-accurate approximation for the traveltimes.

\section{DISCUSSION}

In the previous sections, we have derived the anisotropic extension of the CRS operator and verified it with numerical examples. In this section, we briefly address several points that arose during our investigation. 
Instead of three attributes, namely two wavefront curvature radii and an angle, and the near-surface velocity in the 2-D isotropic zerooffset case, our anisotropic zero-offset operator requires four attributes and a velocity. Although the operator in eq. (20) is expressed in terms of two radii of wavefront curvature, $R_{N}$ and $R_{N I P}$, three angles $\Theta_{0}, \vartheta_{0}, \psi_{0}$, and two velocities $v_{0}, V_{0}$, only three of the latter five quantities are independent. With the relationships

$V_{0}=v_{0} \cos \psi_{0}=v_{0} \cos \left(\vartheta_{0}-\Theta_{0}\right)$

it is possible to express the operator in terms of only three of the above-mentioned five quantities, namely, one velocity and two angles. We have expressed the first-order derivatives in eq. (20) by the phase angle and phase velocity because these describe the slowness. For the second-order terms, we use the ray velocity and angle as well as angle $\psi_{0}$ because these provide the most compact expression.

Most established anisotropic traveltime expressions (e.g. Tsvankin \& Thomsen 1994) consider non-hyperbolic moveout. The corresponding operators are assumed to provide an accurate description of the traveltime over an extended offset range, from zero to far offsets. The non-hyperbolic or anelliptic term that controls the behaviour at large offsets results as a consequence of the presence of both anisotropy and heterogeneity in the subsurface. In the stacking parameter determination, the effects of both anisotropy and heterogeneity are averaged over the entire offset range, that is, globally. Therefore, the non-hyperbolic term alone does not permit to separate the two underlying causes. In contrast, our operators were developed for the application with small midpoint and offset apertures, that is, locally. Instead of describing the traveltimes for the entire offset range with parameters for a single operator, the entire offset range is divided into subranges for which our method provides a local fit with individual parameters for each subrange. Furthermore, taking midpoints into account, too, leads to additional information. If the wavefront attributes are determined with the finite-offset formulation, the local character of the operator could therefore lead to insight for a potential separation of anisotropy and heterogeneity.

As already suggested in the introduction, the determination of all wavefront attributes in the anisotropic case by stacking is not generally possible unless model assumptions are made. For the stack itself, this is not an issue because the shape of the operator is the same as in the isotropic case. If we wish to apply the attributes, for example, for NIP-wave (Duveneck 2004) or diffraction (Bauer et al. 2017) tomography, model assumptions must be made. These assumptions can then be used to calculate the anisotropic attributes from the three parameters obtained during the stack.

Traveltime tomography for anisotropic media has been suggested by, for example, Chapman \& Pratt (1992), Pratt \& Chapman (1992) and Mensch \& Farra (2002). Several authors (e.g. Billette \& Lambaré 1998; Duveneck 2004) have since then shown that including further information in addition to the traveltimes can improve the results of the tomographic inversion: Billette \& Lambaré (1998) use traveltimes and slopes for their stereotomography, whereas Duveneck's (2004) NIP-wave tomography includes also wavefront curvatures. Both methods were originally developed for isotropic media. A detailed comparison can be found in Dümmong et al. (2008). Barbosa et al. (2008) and Nag et al. (2010) extended the stereotomography to anisotropy. The extension of NIP-wave (Duveneck 2004) and diffraction (Bauer et al. 2017) tomography to anisotropy is currently under investigation.

Since the hyperbolic formula is derived by a Taylor expansion it does not distinguish between homogeneous, heterogeneous and anisotropic media. Therefore, data from any such medium can be equally well fitted by the hyperbolic operator as long as we stay within the basic assumption of short spread.

\section{CONCLUSIONS}

We have derived a CRS-type multiparameter operator for anisotropic media in 3-D. The operator is valid for finite-offset configuration including the zero-offset configuration as a special case. It does not require any model assumptions. Numerical examples demonstrate that the accuracy of the operator is of the same order as for isotropic media.

Like its isotropic counterpart, the anisotropic operator is expressed in terms of angles and wavefront curvatures. To account for the anisotropy, the distinction between ray (group) and phase angles and velocities must be considered. In the zero-offset case, the anisotropic operator requires an additional angle that describes the deviation between the group and phase directions. For finite offsets, two additional angles are required.

If no model assumptions are made, only three of the four wavefield attributes in the 2-D zero-offset case can be determined by stacking and semblance analysis because the shape of the operator remains the same as for isotropy. This is an advantage because it means that the stack result does not depend on the presence or absence of anisotropy. If, on the other hand, model assumptions are made, the model provides the necessary relations between the wavefront attributes. In that case, all attributes can be determined by stacking.

In many applications, model assumptions enter only after the stacking. In these further processing steps, neglecting potential anisotropy can lead to errors, for example, if velocity tomography is performed based on isotropic wavefront attributes while the medium is anisotropic. With our new parametrisation, anisotropic wavefront attributes can be applied as soon as an anisotropic model is considered.

Furthermore, we like to think that this new formulation enhances our understanding of surface-measured wavefield properties in arbitrary 3-D anisotropic media. 


\section{ACKNOWLEDGEMENTS}

We are grateful to the members of the Applied Seismics Group in Hamburg for continuous and inspiring discussion, in particular to Benjamin Schwarz, Jan Walda, Sophia Wißmath and Boris Kashtan. Suggestions by Alexey Stovas and an anonymous reviewer are highly appreciated. Further thanks go to Jan Walda for proof-reading and Pam Ehli for support with linguistic issues. Korbinian Sager generated traveltime tables using NORSAR ray modelling software. This work was partially supported by the sponsors of the Wave Inversion Technology (WIT) consortium.

\section{REFERENCES}

Bakhtiari Rad, P., Schwarz, B., Gajewski, D. \& Vanelle, C., 2018. Commonreflection-surface-based prestack diffraction separation and imaging, Geophysics, 83, S47-S55.

Barbosa, B., Costa, J., Gomes, E. \& Schleicher, J., 2008. Resolution analysis for stereotomography in media with elliptic and anelliptic anisotropy, Geophysics, 73, R49-R58.

Bauer, A., Schwarz, B. \& Gajewski, D., 2017, Utilizing diffractions in wavefront tomography, Geophysics, 82, R65-R73.

Baykulov, M. \& Gajewski, D., 2009. Prestack seismic data enhancement with partial common reflection surface (CRS) stack, Geophysics, 74, V49-V58.

Billette, F. \& Lambaré, G., 1998. Velocity macro-model estimation from seismic reflection data by stereotomography, Geophys. J. Int., 135, 671690.

Chapman, C. H. \& Pratt, R. G., 1992. Traveltime tomography in anisotropic media-I. Theory, Geophys. J. Int., 109, 1-19.

Červený, V., 2001. Seismic Ray Theory, Cambridge Univ. Press.

Daley, P. F. \& Hron, F., 1979a. Reflection and transmission coefficients for seismic waves in ellipsoidally anisotropic media, Geophysics, 44, 27-38.

Daley, P. F. \& Hron, F., 1979b. SH waves in layered transversely anisotropic media - an asymptotic expansion approach, Bull. seism. Soc. Am., 69, 689-711.

Dell, S. \& Gajewski, D., 2011. Common-reflection-surface-based workflow for diffraction imaging, Geophysics, 76, S187-S195.

Duveneck, E., 2004. Velocity model estimation with data-derived wavefront attributes, Geophysics, 69, 265-274.

Dümmong, S. \& Gajewski, D., 2008. A multiple suppression method via CRS attributes, in 78th Annual International Meeting, SEG, Expanded Abstracts, pp. 2531-2535.

Dümmong, S., Meier, K., Gajewski, D. \& Hübscher, C., 2008. Comparison of prestack stereotomography and NIP wave tomography for velocity model building: Instances from the Messinian evaporites, Geophysics, 73, VE291-VE302.

Fomel, S. \& Kazinnik, R., 2013. Non-hyperbolic common reflection surface, Geophys. Prosp., 61, 21-27.

Gelchinsky, B., Berkovitch, A. \& Keydar, S., 1999. Multifocusing homeomorphic imaging: Part 1. Basic concepts and formulas, J. Appl. Geophys., 42, 229-242.

Gjøystdal, H., Reinhardsen, J. E. \& Ursin, B., 1984. Traveltime and wavefront curvature calculations in three-dimensional inhomogeneous layered media with curved interfaces, Geophysics, 49, 1466-1494.

Hubral, P. \& Krey, T., 1980. Interval Velocities from Seismic Reflection Time Measurements, SEG Monograph.

Höcht, G., 2002, Traveltime approximations for 2D and 3D media and kinematic wavefield attributes, $P h D$ thesis, University of Karlsruhe.

Mayne, W. H., 1962. Common reflection point horizontal data stacking techniques, Geophysics, 27, 927-938.
Mensch, T. \& Farra, V., 2002. $P$-wave tomography in inhomogeneous orthorhombic media, Pure appl. Geophys., 159, 1855-1879.

Müller, N. A., 2007, The 3D Common-Reflection-Surface stack-theory and application, PhD thesis, University of Karlsruhe.

Müller, T., 1999, The Common Reflection Surface stack method-seismic imaging without explicit knowledge of the velocity model, PhD thesis, University of Karlsruhe.

Nag, S., Alerini, M. \& Ursin, B., 2010. PP/PS anisotropic stereotomography, Geophys. J. Int., 181, 427-452.

Pratt, R. G. \& Chapman, C. H., 1992. Traveltime tomography in anisotropic media - II. Application, Geophys. J. Int., 109, 20-37.

Schwarz, B., Vanelle, C., Gajewski, D. \& Kashtan, B., 2014. Curvatures and inhomogeneities: an improved common reflection surface approach, Geophysics, 79, S231-S240.

Shah, P. M., 1973. Use of wavefront curvature to relate seismic data with subsurface parameters, Geophysics, 38, 812-825.

Spinner, M., 2007, CRS-based minimum-aperture Kirchhoff migration in the time domain, PhD thesis, University of Karlsruhe.

Taner, M. T. \& Koehler, F., 1969. Velocity spectra - digital computer derivation and application of velocity functions, Geophysics, 34, 859-881.

Thomsen, L., 1986. Weak elastic anisotropy, Geophysics, 51, 1954-1966.

Tsvankin, I., 2001. Seismic Signatures and Analysis of Reflection Data in Anisotropic Media, Pergamon.

Tsvankin, I. \& Thomsen, L., 1994. Nonhyperbolic reflection moveout in anisotropic media, Geophysics, 59, 1290-1304.

Tygel, M., Müller, T., Hubral, P. \& Schleicher, J., 1997. Eigenwave-based multiparameter traveltime expansions, in 67th Annual International Meeting, SEG, Expanded Abstracts, pp. 1770-1773.

Ursin, B., 1982. Quadratic wavefront and traveltime approximations in inhomogeneous layered media with curved interfaces, Geophysics, 47, 10121021.

Vanelle, C., Bobsin, M., Schemmert, P., Kashtan, B., Schwarz, B. \& Gajewski, D., 2012. i-CRS: a new multiparameter stacking operator for an/isotropic media, in 82nd Annual International Meeting, SEG, Expanded Abstracts, pp. 1-5.

Vanelle, C. \& Gajewski, D., 2002. Second-order interpolation of traveltimes, Geophys. Prosp., 50, 73-83.

Vanelle, C., Kashtan, B., Dell, S. \& Gajewski, D., 2010. A new stacking operator for curved subsurface structures, in 80th Annual International Meeting, SEG, Expanded Abstracts, pp. 3609-3613.

Walda, J. \& Gajewski, D., 2015. Global optimization of the CRS operator using a genetic algorithm, in 77th EAGE Conference and Exhibition, doi:10.3997/2214-4609.201412956.

Xu, S. \& Stovas, A., 2015. Curvature and anisotropy estimation through the CRS approximation, J. Geophys. Eng., 12, 934-945.

Zhang, Y., Bergler, S. \& Hubral, P., 2001. Common-reflection-surface (CRS) stack for common offset, Geophys. Prosp., 49, 709-718.

\section{APPENDIX: ANISOTROPIC CRS FOR 3-D FINITE OFFSET}

Whereas in 2-D a derivation of the anisotropic operator from the laws of geometry was easily feasible, it is not as simple to visualise in the 3-D case. We can, however, derive the 3-D operator by combining our results from the 2-D situation with the ray method.

In 3-D, we need to distinguish between vectors with two or three components as well as between $2 \times 2$ and $3 \times 3$ matrices. Therefore, we denote two-component vectors and $2 \times 2$ matrices with a bold font, for example, vector $\boldsymbol{a}$ and matrix $\mathbf{A}$. Three-component vectors and $3 \times 3$ matrices are also printed in bold, but carry a hat in addition, for example, $\hat{\boldsymbol{b}}$ and matrix $\underline{\mathbf{B}}$. Scalars are printed in regular font. Lower case indices $i, j$ take values $1,2,3$, whereas upper case indices $I, J$ take values 1,2 . 
In general, the operator will retain the same form as in 2-D. We need to replace the scalars describing coordinates and slownesses with vectors and the scalar wavefront curvatures with matrices. For our derivation, we begin with the hyperbolic traveltime expression in source and receiver coordinates suggested in Vanelle \& Gajewski (2002),

$t^{2}(\boldsymbol{s}, \boldsymbol{g})=\left(t_{0}+\boldsymbol{p}_{\boldsymbol{g}} \boldsymbol{\Delta} \boldsymbol{g}^{\top}-\boldsymbol{p}_{\boldsymbol{s}} \boldsymbol{\Delta} \boldsymbol{s}^{\top}\right)^{2}+t_{0}\left(\boldsymbol{\Delta} \boldsymbol{g} \underline{\mathbf{G}} \boldsymbol{\Delta} \boldsymbol{g}^{\top}-\boldsymbol{\Delta} \underline{\boldsymbol{S}} \underline{\boldsymbol{\Delta}} \boldsymbol{s}^{\top}-2 \boldsymbol{\Delta} \underline{\mathbf{N}} \boldsymbol{\Delta} \boldsymbol{g}^{\top}\right)$,

where $\Delta \boldsymbol{s}=\boldsymbol{s}-\boldsymbol{s}_{\mathbf{0}}$ and $\boldsymbol{\Delta} \boldsymbol{g}=\boldsymbol{g}-\boldsymbol{g}_{\mathbf{0}}$ are the distance vectors of the source and receiver positions to the expansion point, respectively, and $t_{0}$ is the traveltime in the expansion point, that is, $t_{0}=t\left(\mathbf{s}_{\mathbf{0}}, \boldsymbol{g}_{\mathbf{0}}\right)$. The coefficients in eq. (A1) are the first- and second-order derivatives of the traveltime with respect to source and receiver coordinates. Namely, the first-order derivatives are the horizontal slowness components, that is,

$\boldsymbol{p}_{\boldsymbol{s}}^{\top}=-\frac{\sin \Theta_{s}}{V_{s}}\left(\begin{array}{c}\cos \Phi_{s} \\ \sin \Phi_{s}\end{array}\right)$ and $\quad \boldsymbol{p}_{\boldsymbol{g}}^{\top}=\frac{\sin \Theta_{g}}{V_{g}}\left(\begin{array}{c}\cos \Phi_{g} \\ \sin \Phi_{g}\end{array}\right)$,

where the angles $\Phi_{s}$ and $\Phi_{g}$ describe the azimuth of the slowness vectors. The second-order derivatives are given by

$S_{I J}=-\left.\frac{\partial^{2} t}{\partial s_{I} \partial s_{J}}\right|_{s_{0}, g_{0}}, \quad G_{I J}=\left.\frac{\partial^{2} t}{\partial g_{I} \partial g_{J}}\right|_{s_{0}, g_{0}} \quad, \quad$ and $\quad N_{I J}=-\left.\frac{\partial^{2} t}{\partial s_{I} \partial g_{J}}\right|_{s_{0}, g_{0}}$.

Note that the $2 \times 2$ matrices $\underline{\mathbf{S}}$ and $\underline{\mathbf{G}}$ are symmetric, while $\underline{\mathbf{N}}$ is not symmetric. Rewriting eq. (A1) into midpoint and half-offset coordinates leads to

$$
\begin{aligned}
t^{2}\left(\boldsymbol{x}_{\boldsymbol{m}}, \boldsymbol{h}\right)= & {\left[t_{0}+\left(\boldsymbol{p}_{\boldsymbol{g}}-\boldsymbol{p}_{s}\right) \boldsymbol{\Delta} \boldsymbol{x}_{\boldsymbol{m}}^{\top}+\left(\boldsymbol{p}_{g}+\boldsymbol{p}_{\boldsymbol{s}}\right) \boldsymbol{\Delta} \boldsymbol{h}^{\top}\right]^{2}+t_{0} \boldsymbol{\Delta} \boldsymbol{x}_{\boldsymbol{m}}\left[\underline{\mathbf{M}}_{g}^{C \boldsymbol{O}}-\underline{\mathbf{M}}_{s}^{C \boldsymbol{O}}\right] \boldsymbol{\Delta} \boldsymbol{x}_{\boldsymbol{m}}^{\top} } \\
& +t_{0} \boldsymbol{\Delta} \boldsymbol{h}\left[\underline{\mathbf{M}}_{g}^{C M P}-\underline{\mathbf{M}}_{s}^{C M P}\right] \boldsymbol{\Delta} \boldsymbol{h}^{\top}+2 t_{0} \boldsymbol{\Delta} \boldsymbol{x}_{\boldsymbol{m}}\left[\underline{\mathbf{M}}_{g}^{C M P}+\underline{\mathbf{M}}_{s}^{C M \boldsymbol{P}}\right] \boldsymbol{\Delta} \boldsymbol{h}^{\top},
\end{aligned}
$$

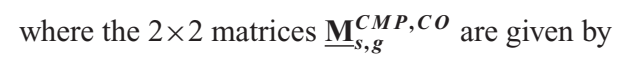

$$
\begin{gathered}
\underline{\mathbf{M}}_{g}^{C o}=\underline{\mathbf{G}}-\underline{\mathbf{N}}, \quad \underline{\mathbf{M}}_{s}^{C o}=\underline{\mathbf{S}}+\underline{\mathbf{N}}^{\top} \\
\underline{\mathbf{M}}_{g}^{C M P}=\underline{\mathbf{G}}+\underline{\mathbf{N}}^{\top}, \underline{\mathbf{M}}_{s}^{C M P}=\underline{\mathbf{S}}-\underline{\mathbf{N}} .
\end{gathered}
$$

Furthermore, eq. (A5) yields

$\underline{\mathbf{M}}_{g}^{C M P}+\underline{\mathbf{M}}_{s}^{C M P}=\underline{\mathbf{M}}_{g}^{C O}+\underline{\mathbf{M}}_{s}^{C O}$.

Note that no assumption on the type of anisotropy was made for the derivation of eq. (A4). It is, therefore, suited to describe seismic traveltimes in arbitrarily anisotropic media, including traveltimes of converted waves.

In our 2-D derivation, we related the second-order derivatives to wavefront curvatures. We will do the same for the 3-D case. Instead of the scalars $K_{s, g}^{C M P, C O}=1 / R_{s, g}^{C M P, C O}$ in 2-D we now consider wavefront curvature matrices $\underline{\mathbf{K}}_{s, g}^{C M P, C \boldsymbol{O}}$ (Červený 2001). Since the following considerations apply to all matrices $\underline{\mathbf{M}}_{s, g}^{C M P, C \boldsymbol{O}}$ as well as $\underline{\mathbf{K}}_{s, g}^{C M P, C \boldsymbol{O}}$, we will from now on omit the superscripts and subscripts indicating the type of experiment and position and refer to these only by $\underline{\mathbf{M}}$ and $\underline{\mathbf{K}}$. Following, for example, Höcht (2002) or Müller (2007), the matrices $\underline{\mathbf{M}}$ and $\underline{\mathbf{K}}$ are related by

$\underline{\mathbf{M}}=\frac{1}{u} \underline{\mathbf{H}} \underline{\mathbf{K}}^{\top}$.

In eq. (A7), $\underline{\mathbf{K}}$ is a wavefront curvature matrix in ray-centred coordinates (according to Müller 2007) or wavefront-orthonormal coordinates (according to Höcht 2002). In ray-centred coordinates (rcc), the unit vector $\hat{\boldsymbol{e}}_{3}^{r c c}$ is given by the ray direction, that is,

$\hat{\boldsymbol{e}}_{3}^{r c c}=\frac{\hat{\boldsymbol{v}}}{|\hat{\boldsymbol{v}}|}$

whereas in wavefront-orthonormal coordinates (woc)

$\hat{\boldsymbol{e}}_{3}^{w o c}=\frac{\hat{\boldsymbol{p}}}{|\hat{\boldsymbol{p}}|}$

that is, the unit vector $\hat{\boldsymbol{e}}_{3}^{\text {woc }}$ points in the direction of the slowness. Matrix $\underline{\mathbf{H}}$ is the upper left $2 \times 2$ submatrix of the transformation matrix from either of these coordinates to the acquisition system. The quantity $u$ is a near-surface velocity that is not further specified.

Only if the medium under consideration is isotropic, we do not need to distinguish between phase and ray (group) velocity because in this case $u=v=V$. Furthermore, wavefront-orthonormal and ray-centred coordinates coincide. This is, however, not the case in anisotropic media, where the according transformation cannot be achieved by a simple $2 \times 2$ matrix. A quick look at the $2-\mathrm{D}$ situation illustrates this: In the main text, we have shown that

$M=\frac{K \cos ^{2} \vartheta}{v \cos ^{3} \psi}=\frac{K \cos ^{2} \vartheta}{V \cos ^{2} \psi}$ 
where $R$ was substituted with $1 / K$. If $\underline{\mathbf{H}}$ described the transformation between acquisition and ray-centred coordinates, eq. (A7) would lead to

$M=\frac{K \cos ^{2} \vartheta}{v}$

with $u=v$ or

$M=\frac{K \cos ^{2} \vartheta}{V}=\frac{K \cos ^{2} \vartheta}{v \cos \psi}$

with $u=V$. Similarly, if $\underline{\mathbf{H}}$ described the transformation between acquisition and wavefront-orthonormal coordinates, we would obtain

$M=\frac{K \cos ^{2} \Theta}{v}$

with $u=v$ or

$M=\frac{K \cos ^{2} \Theta}{V}=M=\frac{K \cos ^{2} \Theta}{v \cos \psi}$

with $u=V$. Whereas these solutions coincide in the special case of isotropy where $\psi=0$, neither one yields the correct result A10 in the anisotropic situation.

In order to find a correct representation for anisotropic media, we refer to Červený (2001) for the following considerations.

The relationship between a matrix $\underline{\mathbf{M}}^{\text {woc }}$ and the corresponding wavefront curvature matrix $\underline{\mathbf{K}}$ is given by

$\underline{\mathbf{K}}=V \underline{\mathbf{M}}^{w o c}$,

where $V$ denotes (as before) the phase velocity and $\underline{\mathbf{M}}^{\text {woc }}$ is the upper left $2 \times 2$ submatrix of the $3 \times 3$ matrix $\underline{\hat{\mathbf{M}}}^{\text {woc }}$ that is defined as the Hessian of the traveltime in wavefront-orthonormal coordinates $x_{i}^{w o c}$,

$\hat{M}_{i j}^{w o c}=\frac{\partial^{2} t}{\partial x_{i}^{w o c} \partial x_{j}^{w o c}}$,

or

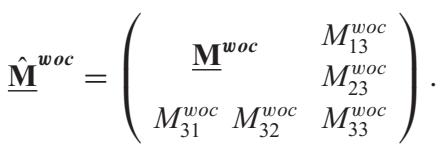

Expressed by the curvature matrix $\underline{\mathbf{K}}$ and the ray velocity vector in wavefront-orthonormal coordinates, $\boldsymbol{v}^{\text {woc }}$, for the locally homogeneous medium we consider matrix $\underline{\hat{\mathbf{M}}}^{\text {woc }}$ becomes

$\underline{\hat{\mathbf{M}}}^{\text {woc }}=\frac{1}{V^{3}}\left(\begin{array}{cc}V^{2} \underline{\mathbf{K}} & -V v_{J}^{w o c} M_{1 J}^{w o c} \\ -V v_{I}^{w o c} M_{I 1}^{w o c}-V v_{I}^{w o c} M_{I 2}^{w o c} & -V v_{J}^{w o c} M_{2 J}^{w o c} v_{I}^{w o c} v_{J}^{w o c} M_{I J}^{w o c}\end{array}\right)$,

where summation convention is applied. The latter relationship can be derived from the definition of the slowness vector $\hat{\boldsymbol{p}}$ as the gradient of the traveltime $t$ and the fact that the scalar product of the group velocity and slowness vectors equals one in any coordinate system, that is,

$\hat{\boldsymbol{p}} \cdot \hat{\boldsymbol{v}}=\vec{\nabla} t \cdot \hat{\boldsymbol{v}}=1$.

Assuming a locally homogeneous medium and considering wavefront-orthonormal coordinates, where $x_{i}=x_{i}^{w o c}$, eq. (A19) becomes

$\frac{\partial t}{\partial x_{1}^{w o c}} v_{1}^{w o c}+\frac{\partial t}{\partial x_{2}^{w o c}} v_{2}^{w o c}+\frac{\partial t}{\partial x_{3}^{w o c}} v_{3}^{w o c}=1$.

The gradient of this expression is zero, which leads to

$M_{i j}^{w o c} v_{i}^{w o c}=0 \quad$ and

$M_{i j}^{w o c} v_{j}^{w o c}=0$,

which, in turn, and with $v_{3}^{w o c}=V$ provides eq. (A18).

With the transformation matrix $\underline{\hat{\mathbf{H}}}$ from wavefront-orthonormal coordinates to the acquisition system,

$\hat{H}_{i j}=\frac{\partial x_{i}}{\partial x_{j}^{w o c}}=\frac{\partial x_{j}^{w o c}}{\partial x_{i}}$,

we can now determine the $3 \times 3$ Hessian matrix of the traveltimes, $\underline{\hat{\mathbf{M}}}$, in the acquisition system with $\hat{\boldsymbol{e}}_{3}$ oriented in $z$-direction by

$\underline{\hat{\mathbf{M}}}=\underline{\hat{\mathbf{H}}} \underline{\hat{\mathbf{M}}}^{\text {woc }} \underline{\hat{\mathbf{H}}}^{\top}$.

Applied to each experiment (CMP, CO) at the source and receiver coordinates, the upper left $2 \times 2$ submatrices of the according matrices $\underline{\hat{\mathbf{M}}}$ are the sought-after matrices $\underline{\mathbf{M}}$ in the operator A4. 


\section{A1 Reduction to the 2-D case}

For illustration, we show that this procedure leads to the correct result in the 2-D case. With the transformation matrix

$\underline{\mathbf{H}}=\left(\begin{array}{rr}\cos \Theta & \sin \Theta \\ -\sin \Theta & \cos \Theta\end{array}\right)$

and the ray velocity vector in the acquisition and wavefront-orthonormal coordinates,

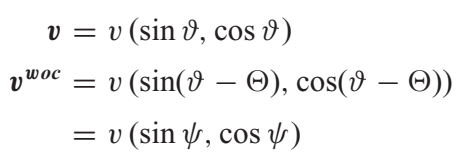

the matrix $\underline{\mathbf{M}}^{\text {woc }}$ becomes

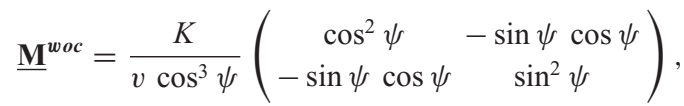

where $V=v \cos \psi$ was substituted. After carrying out the coordinate transformation, we find that

$$
\begin{aligned}
M & =\frac{K}{v \cos ^{3} \psi}\left(\cos ^{2} \Theta \cos ^{2} \psi+\sin ^{2} \Theta \sin ^{2} \psi-2 \sin \Theta \sin \psi \cos \Theta \cos \psi\right) \\
& =\frac{K}{v \cos ^{3} \psi}(\cos \Theta \cos \psi-\sin \Theta \sin \psi)^{2} \\
& =\frac{K \cos ^{2}(\Theta+\psi)}{v \cos ^{3} \psi} \\
& =\frac{K \cos ^{2} \vartheta}{v \cos ^{3} \psi} .
\end{aligned}
$$

This is the correct result that we also obtained from the 2-D derivation. 